MS44-04

\section{Kinetics of gas sorption by porous frameworks probed by sub-second synchrotron powder X-ray diffraction}

Iurii Dovgaliuk$^{1}$, Vadim Diadkin ${ }^{1}$, Yaroslav Filinchuk², Dmitry Chernyshov ${ }^{1}$

1. Swiss-Norwegian Beamlines at the European Synchrotron Radiation Facility, Grenoble, France

2. Université catholique de Louvain, Louvain-la-Neuve, Belgium

email: iurii.dovgaliuk@esrf.fr

Numerous porous frameworks have been intensively investigated as promising materials for gas storage and separation, sensing, catalysis, magnetism, luminescence, drug delivery and other applications.[1] To date, the dynamic behavior and involved mechanisms of flexible porous materials still mostly rationalized by the structural differences between the thermodynamically stable structures of the initial (empty) and final (filled) states or by following adsorption at equilibrium conditions.[2, 3] Very often fascinating phenomena upon gas adsorption can be either not well-understood (e.g. the switching of the gas selectivity),[4] or missed out without in situ temperature/pressure variable experiments (e.g. negative gas adsorption).[5] The time-resolved observation of structural evolution during guest uptake may shed light on actual mechanisms, kinetic and thermodynamic parameters of the sorption processes.

In this work, we present the result of a kinetic experiment, where the adsorption of noble gases by nanoporous $\gamma-\mathrm{Mg}\left(\mathrm{BH}_{4}\right)_{2}$ has been probed via sub-second powder diffraction of synchrotron radiation. The combined analysis of fluorescent background and structural information from sequential Rietveld analysis has uncovered 3 time-dependent processes related to gas uptake and provided a quantitative characterization in terms of rates, kinetic models and activation energies. Adsorption starts form the gas uptake by initial $\gamma-\mathrm{Mg}\left(\mathrm{BH}_{4}\right)_{2}$ host structure and then followed by a formation of secondary phase with higher kinetic barrier. The third process was observed at high temperatures and long exposure time and can be tentatively associated with radiation damage of the sample.

Our result shows the importance of diffraction methods for the characterization of thermodynamics and kinetics of gas adsorption by porous frameworks; the used approach can be further developed for more complicated in situ experimentation, where diffraction data would help to untangle different processes superimposed in macroscopic probes.

References:

[1] K. Adil et al. Chem. Soc. Rev. 2017, 46, 3402-3430; [

[2]] Zhang J.-P. et al. Chem. Soc. Rev. 2014, 43, 5789-5814;

[3] I. Dovgaliuk et al. Chem. Eur. J. 2017, 70, 17714-17720;

[4] Fernandez, C. A. et al. J. Am. Chem. Soc. 2012, 134, 9046-9049;

[5] a) Stoeck U. et al. Chem. Commun. 2012, 48, 10841-10843; b)

Krause, S. et al. Nature 2016, 532, 348-352.

Keywords: kinetics, gas sorption, powder X-ray diffraction

\section{MS44-05}

\section{Combining experimental and computational techniques to understand phase transitions of nucleobase adenine}

Dubravka Sisak Jung ${ }^{1}$, I. Halasz ${ }^{2}$, D. McDonagh ${ }^{3}$, G.M. Day ${ }^{3}$

1. DECTRIS, Switzerland

2. Division of Physical Chemistry, Ruđer Bošković Institute, Croatia

3. School of Chemistry, University of Southampton, United

Kingdom

email: dubravka.sisak@dectris.com

Monitoring phase transitions in-situ becomes increasingly difficult if the transitions feature a series of similar structures, and if the occurring phases cannot be isolated from the mixture. In these cases, complete powder X-ray diffraction (PXRD) need to be collected in a minimal number of steps and exhibit statistics and resolution that allow for structure determination and refinement. While these problems are successfully addressed at synchrotron beamlines, laboratory diffractometers are rarely fit for the task. One of the challenging samples is adenine, a small and rigid molecule that exhibits two polymorphs: one monoclinic (I) [1] and the other orthorhombic (II) [2]. Both polymorphs feature a layered structure, what makes their PXRD patterns similar and difficult to resolve when in a mixture. Both phases are bench-stable at room temperature, with polymorph IIbeing metastable, while at temperatures above $280{ }^{\circ} \mathrm{C}$ phase (I) transforms into (II) [2].

In this work, adenine phase transition is monitored in-situ using a laboratory diffractometer in the Debye-Scherrer geometry, equipped with a Mo source, a specially developed oven and two simultaneously operating detectors. In this way, $74^{\circ}$ in $2 \theta$ of high-resolution data could be collected in only two shots. The resulting PXRD patterns revealed two additional adenine phases: IIIand $\mathbf{X}$. The phase III was obtained pure at temperatures higher than $350{ }^{\circ} \mathrm{C}$. It was characterized as a disordered layered structure using the DISCUS program. At about $200{ }^{\circ} \mathrm{C}$, several low-intensity peaks indicated a new phase, $(\mathbf{X})$. Although these peak positions could be indexed with a triclinic cell of a favorable volume, the data did not allow for cell validation or refinement. To complement the diffraction study, crystal structure prediction was carried out for adenine using quasi-random sampling in a large number of space groups. This has generated a set of structures energetically close to the two known polymorphs. A few of these computer-generated structures could correspond to the phase $(\mathbf{X})$ observed in the PXRD patterns. Calculations are ongoing.

\section{References:}

[1] Mahapatra, S.; Nayak, S. K.; Prathapa, S. J.; Guru Row, T. N. Cryst. Growth Des. 2008, 8, 1223.

[2] Stolar T. et al. Cryst. Growth Des. 2016, 16, 3262.

Keywords: in situ, X-ray powder diffraction, structure prediction 